\title{
Deeply penetrating photoacoustic tomography in biological tissues enhanced with an optical contrast agent
}

\author{
Geng Ku and Lihong V. Wang \\ Optical Imaging Laboratory, Department of Biomedical Engineering, 3120 TAMU, Texas A\&M University, \\ College Station, Texas 77843-3120 \\ Received September 15, 2004

\begin{abstract}
Photoacoustic tomography (PAT) in a circular scanning configuration was developed to image deeply embedded optical heterogeneity in biological tissues. While the optical penetration was maximized with near-infrared laser pulses of 800-nm wavelength, the optical contrast was enhanced by Indocyanine Green (ICG) dye whose absorption peak matched the laser wavelength. This optimized PAT was able to image objects embedded at depths of as much as $5.2 \mathrm{~cm}, 6.2$ times the 1/e optical penetration depth, in chicken breast muscle at a resolution of $<780 \mu \mathrm{m}$ and a sensitivity of $<7 \mathrm{pmol}$ of ICG in blood. The resolution was found to deteriorate slowly with increasing imaging depth. The effects of detection bandwidth on the quality of images acquired simultaneously by four different ultrasonic transducers are described. () 2005 Optical Society of America

OCIS codes: 170.5120, 170.1470, 170.3880, 170.0100.
\end{abstract}

Photoacoustic tomography (PAT), which is based on the measurement of laser-induced ultrasonic waves, is a nonionizing imaging modality for visualizing biological tissues with high optical contrast and high ultrasonic resolution. This imaging modality has been successfully applied to imaging vasculature structures and tumor angiogenesis a few millimeters beneath the skin. ${ }^{1-3}$ Oraevsky and colleagues studied the imaging sensitivity of PAT on deeply embedded objects, using tissue phantoms ${ }^{4}$ and they reported a PAT image of a ductal carcinoma at a depth of $1.1 \mathrm{~cm}$ from the surface of breast tissue. ${ }^{5,6}$ Because of the overwhelming scattering effect of light in biological tissues, the intensity of light, and hence the photoacoustic signal-to-noise ratio (SNR), decreases with depth exponentially with a decay constant of a few millimeters. The attenuation in the intensity of light can be minimized by careful choice of the excitation laser wavelength such that the optical penetration in the tissues is maximized. The near infrared has a low absorption coefficient and a relatively low scattering coefficient in biological tissues and can, consequently, provide deep penetration at the expense of reduced photoacoustic signal strength. However, photoacoustic signals can be enhanced by use of a matched optical contrast agent. ${ }^{7}$ Indocyanine Green (ICG) dye has a strong absorption peak in the near infrared and can serve such a purpose. ICG is widely used in clinical applications such as cardiac output monitoring, hepatic function studies, ${ }^{9}$ angiography in ophthalmology ${ }^{10}$ and tumor detection. ${ }^{11}$ In this Letter we report deeply penetrating PAT that was based on an optimal optical wavelength and a matched optical contrast agent.

In Fig. 1 our experimental setup for deeply penetrating PAT is shown. A pulsed Ti:sapphire laser (Lotis TII; Symphotic), which is pumped by a $Q$-switched Nd:YAG laser at a repetition rate of $10 \mathrm{~Hz}$, provides $15-\mathrm{ns}$ near-infrared laser pulses at a wavelength of $800 \mathrm{~nm}$. The laser beam is expanded by a concave lens, homogenized by ground glass, and then directed onto a sample made from chicken breast muscle tissue. The incident laser energy density on the tissue surface is $\sim 7 \mathrm{~mJ} / \mathrm{cm}^{2}$, which is less than the "maximum permissible exposure"12 of $32 \mathrm{~mJ} / \mathrm{cm}^{2}$ for human skin at this wavelength. The PAT system has four independent detection channels that can receive photoacoustic signals through multiple ultrasonic transducers simultaneously. Four ultrasonic transducers (V323/2.25-MHz, V383/3.5-MHz, V157/5-MHz, and V312/10-MHz; Panametrics), with nominal bandwidths that range from 50\% to $80 \%$ of their specified central frequencies, are used for detection in four different frequency ranges. The diameters of their active elements are 0.6, 1, 0.3, and $0.6 \mathrm{~cm}$ for the 2.25-, 3.5-, 5-, and $10-\mathrm{MHz}$ transducers, respectively. The $3.5-\mathrm{MHz}$ transducer is cylindrically focused in the vertical dimension with a focal length of $3.2 \mathrm{~cm}$; the others have flat active surfaces. The transducers convert the photoacoustic pressure into piezoelectric signals. The signals are subsequently amplified by amplifiers (ZFL-500LN, Mini-Circuits and 5072PR, Panametrics), bandpass filtered by our recently homemade filters, and finally recorded by an oscilloscope (TDS-540A; Tektronix). The sample and the transducers are immersed in a tank of water for coupling of the photoacoustic waves to the tranducers. The transducers are driven by a stepper motor to scan horizontally along a 10 -cm-diameter circle about

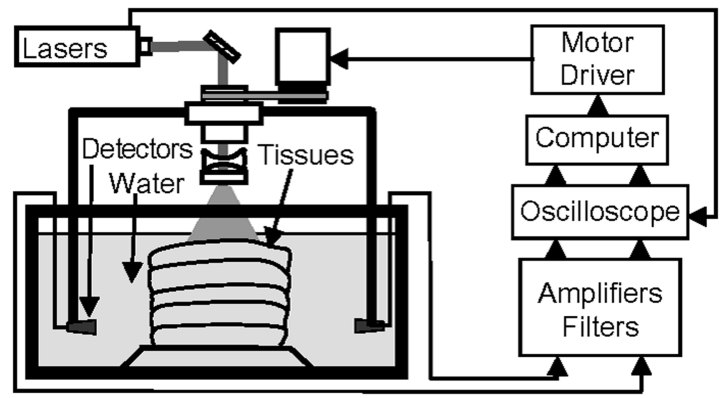

Fig. 1. Experimental setup for deeply penetrating PAT. 
the sample in steps of $1.5^{\circ}$. A personal computer is used to control the scanning and data acquisition.

Three pairs of transparent plastic tubes of $300-\mu \mathrm{m}$ inner diameter each were embedded in chicken breast tissues of $\sim 7.5-\mathrm{cm}$ diameter in their transverse cross sections [Fig. 2(a)]. The first pair of tubes was filled with anticoagulation-treated whole rat blood; the second, with ICG solution in distilled water $(323 \mu \mathrm{M})$; the last, with ICG in rat blood $(129 \mu \mathrm{M})$. Those centripetally arranged objects were placed in the transducers' scanning plane and covered by chicken breast tissues of various thicknesses [Fig. 2(b)]. At 800-nm wavelength, the optical absorption coefficient is approximately $4-5 \mathrm{~cm}^{-1}$ for whole blood, $16 \mathrm{~cm}^{-1}$ for ICG in water, and $43 \mathrm{~cm}^{-1}$ for ICG in blood. ${ }^{13}$ When they are excited by laser light, the embedded objects emit photoacoustic waves that propagate through the medium. The photoacoustic signals are picked up by the circularly stationed transducers and fed into the image reconstruction based on a backprojection algorithm. ${ }^{14}$

The tissue's cross section containing the embedded objects was imaged when four pieces of chicken breast tissue were sequentially stacked to make the embedded objects $1.3,2.6,4.2$, and $5.2 \mathrm{~cm}$ deep from the laserilluminated tissue surface. Figures 2(c)-2(f) show the photoacoustic images obtained at these depths by the $2.25-\mathrm{MHz}$ transducer. A dust grain in the imaging plane is also clearly imaged. The two whole-blood tubes are liminal at an imaging depth of $1.3 \mathrm{~cm}$ and became unrecognizable when the depth increased further. The ICG solution in water is discernible at depths of as much as $5.2 \mathrm{~cm}$. The two ICGenhanced blood tubes have the strongest photoacoustic signals and are clearly imaged throughout. As the photoacoustic strength is proportional to the absorbed optical energy density in an optical absorber, the photoacoustic signal strengths of the three kinds of object at the same depth reflect the differences in their optical absorption properties. To improve the image quality we averaged the photoacoustic signals 50 times at a depth of $1.3 \mathrm{~cm}, 100$ times at $2.6 \mathrm{~cm}$, and 400 times each at 4.2 and $5.2 \mathrm{~cm}$.

At each depth, four photoacoustic images were simultaneously acquired by the four transducers. Figures 2(f), 2(g), and 2(h) show the images of the ICG-enhanced tubes at the $5.2-\mathrm{cm}$ depth by the $2.25-$, $3.5-$, and $5-\mathrm{MHz}$ transducers, respectively. The $10-\mathrm{MHz}$ transducer failed to provide a clear image at this depth. The samples are well delineated in all three images, with slight differences in the details, which can be accounted for by the transducers' bandwidths and geometry. ${ }^{3}$ The image from the $2.25-\mathrm{MHz}$ transducer has the best SNR, whereas the image from the $5.0-\mathrm{MHz}$ transducer provides the sharpest image profile. Because there is more photoacoustic energy in the lower frequency range, the $2.25-\mathrm{MHz}$ transducer receives stronger signals than the other transducers; in addition, its larger active area helps to increase its detection sensitivity. However, a larger aperture yields a poorer off-center resolution.

The light intensities that transmit through the four tissue thicknesses are detected with a photodiode de- tector (DET110 from Thorlabs; Fig. 3). The effective attenuation coefficient of the chicken breast tissue is fitted to be $1.2 \mathrm{~cm}^{-1}$ based on Beer's law. Therefore the 5.2-cm maximum imaging depth amounts to approximately 6.2 times the $1 / e$ optical penetration depth, corresponding to a 27-dB attenuation from the incident to the transmitted optical energy density.

The image width of each tube is also examined in relation to the imaging depth (Fig. 3). Inspecting the photoacoustic images at each depth shown in Fig. 2, first we identified the dark lines for the tubes containing blood and ICG, and then we calculated the zero-crossing width of the reconstructed image profile across each tube as the image width. ${ }^{3}$ The variation in the image width reflects the change in the in-plane imaging resolution. The means and standard deviations of the image width within a segment of $5 \mathrm{~mm}$ in the central area along the tube were calculated. When the tissue thickness increased, the image width remained near $580 \mu \mathrm{m}$ within the first $\sim 4-\mathrm{cm}$ imaging depth and then slowly became wider, increasing to $\sim 780 \mu \mathrm{m}$ at a depth of $5.2 \mathrm{~cm}$. Therefore we can conclude that the in-plane spatial resolution is better than $780 \mu \mathrm{m}$ at

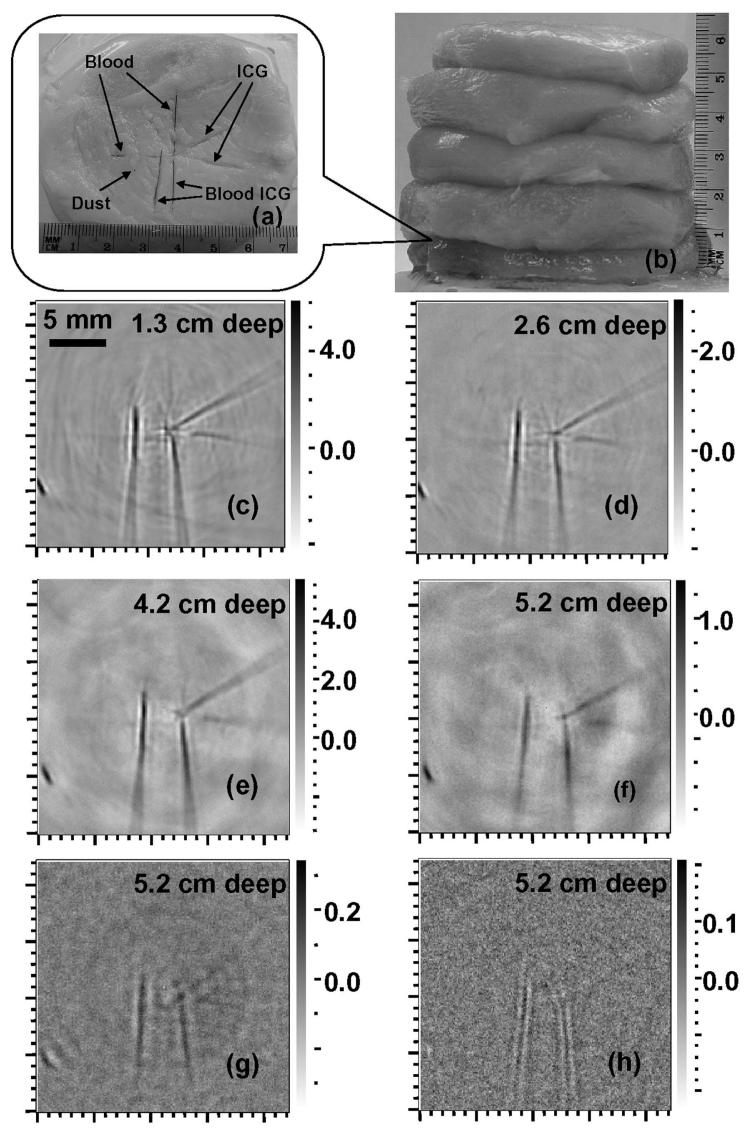

Fig. 2. Two-dimensional photoacoustic imaging at various depths. Photographs of (a) the cross section of chicken breast tissue in which objects containing blood, ICG, or both are embedded and (b) the entire sample. Twodimensional photoacoustic images acquired with a $2.25-\mathrm{MHz}$ ultrasonic transducer at depths of (c) 1.3, (d) 2.6 , (e) 4.2 , and (f) $5.2 \mathrm{~cm}$. Two-dimensional photoacoustic images at a $5.2-\mathrm{cm}$ depth acquired by (g) $3.5-$ and (h) $5.0-\mathrm{MHz}$ ultrasonic transducers. 


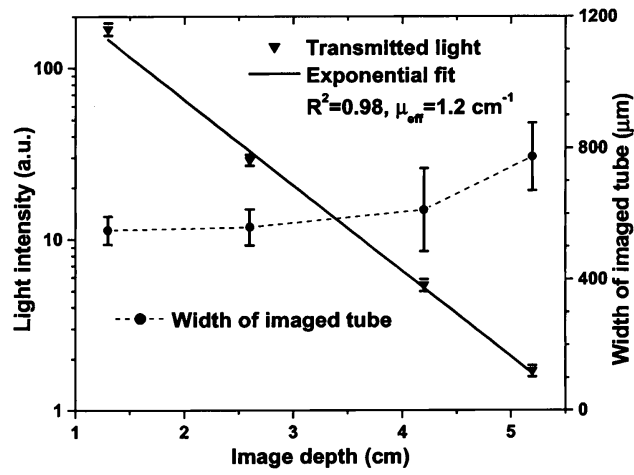

Fig. 3. Transmitted laser light intensity and image width of the tube containing blood and ICG in the photoacoustic images acquired by the $2.25-\mathrm{MHz}$ ultrasonic transducer versus the depth of the imaged cross section in the chicken breast tissue.

the 5.2-cm imaging depth. One can measure the true resolution more accurately by imaging a much smaller object. Theoretically, the resolution is determined by the spectra of the received photoacoustic signals and the SNR. The photoacoustic spectra, which depend on the excitation laser pulse profile and the tissue structures in the imaging plane as well as on the detection bandwidth, remain unchanged when the tissue thickness increases. When the SNR is sufficiently high, the resolution is insensitive to increased tissue thickness. However, when the SNR is low enough to narrow the useful spectra of the signals above the noise level, the resolution decreases with increasing tissue thickness. Of course, the off-plane spatial resolution (slice thickness) is determined by the aperture of the active element in the ultrasonic transducer if the transducer is unfocused or by the numerical aperture of the transducer if the transducer is focused.

We can estimate the sensitivity of our PAT on the detection of ICG based on the ICG concentration and the resolvable sample volume. At the $5.2-\mathrm{cm}$ imaging depth the resolvable sample volume is $\sim\left(\pi \times 0.3^{2} / 4\right) \times$ $0.78 \mathrm{~mm}^{3}$, where $0.3 \mathrm{~mm}$ is the diameter of the tubes and $0.78 \mathrm{~mm}$ is the overestimated resolution. Based on the concentration of ICG in the blood-ICG mixture, the resolvable sample volume contains $\sim 7 \mathrm{pmol}$ of ICG. Because the spatial resolution is better than $0.78 \mathrm{~mm}$, the sensitivity is actually better than $7 \mathrm{pmol}$. Of course, the sensitivity is even better at shallower imaging depths. If the incident laser energy density is increased toward the maximum permissible exposure, the sensitivity can be further improved.

In summary, three aspects of photoacoustic tomography were optimized to image deep tissue struc- tures. First, near-infrared laser light, which can deeply penetrate biological tissues, was employed as the excitation source; second, an optical contrast agent, Indocyamine Green dye, was applied to enhance the optical absorption of blood; third, multiple ultrasonic transducers were simultaneously used to balance detection sensitivity and image resolution. It has been demonstrated that fine tubes that contain blood and ICG embedded at depths greater than $5 \mathrm{~cm}$ in chicken breast tissue can be clearly imaged by PAT with better than $780-\mu \mathrm{m}$ resolution. These results suggest that a sample as much as $10 \mathrm{~cm}$ thick could be imaged if both sides of the sample were illuminated. Therefore nonionizing PAT can potentially image deep vasculatures in the breast and other organs.

We thank G. Stoica for assistance with blood samples and X. Xie, X. Wang, M. Sivaramakrishnan, and $\mathrm{K}$. Song for laboratory assistance. This study is sponsored in part by National Institutes of Health grants R01 EB000712 and R01 NS46214 and by Texas Advanced Technology Program grant 000512-0063-2001. L. V. Wang's e-mail address is lwang@tamu.edu.

\section{References}

1. R. G. M. Kolkman, E. Hondebrink, W. Steenbergen, and F. F. M. de Mul, IEEE J. Sel. Top. Quantum Electron. 9, 343 (2003).

2. X. Wang, Y. Pang, G. Ku, X. Xie, G. Stoica, and L.-H. V. Wang, Nat. Biotechnol. 21, 803 (2003).

3. G. Ku, X. Wang, X. Xie, G. Stoica, and L.-H. V. Wang, Phys. Med. Biol. 49, 1329 (2004).

4. R. O. Esenaliev, A. A. Karabutov, and A. A. Oraevsky, IEEE J. Sel. Top. Quantum Electron. 5, 981 (1999).

5. A. A. Karabutov, E. V. Savateeva, and A. A. Oraevsky, Laser Phys. 13, 711 (2003).

6. A. A. Oraevsky, E. V. Savateeva, S. V. Solomatin, A. A. Karabutov, V. G. Andreev, Z. Gatalica, T. Khamapirad, and P. M. Henrichs, Proc. SPIE 4618, 81 (2002).

7. X. Wang, G. Ku, M. A. Wegiel, D. J. Bornhop, G. Stoica, and L.-H. V. Wang, Opt. Lett. 29, 730 (2004).

8. Y.-L. He, H. Tanigami, H. Ueyama, T. Mashimo, and I. Yoshiya, Crit. Care Med. 26, 1446 (1998).

9. J. Caesar, S. Shaldon, L. Chiandussi, L. Guevara, and S. Sherlock, Clin. Sci. 21, 43 (1961).

10. B. F. Hochheim, Arch. Ophthalmol. 86, 564 (1971).

11. M. M. Haglund, M. S. Berger, and D. W. Hochman, Neurosurgery 38, 308 (1996).

12. American National Standards Institute, "American National Standard for the Safe Use of Lasers," Standard Z 136.1-2000 (American National Standards Institute, Washington, D.C., 2000).

13. M. L. J. Landsman, G. Kwant, G. A. Mook, and W. G. Zijlstra, J. Appl. Physiol. 40, 575 (1976).

14. M. Xu and L.-H. V. Wang, IEEE Trans. Med. Imag. 21, 814 (2002). 